PANDORA'S BOX

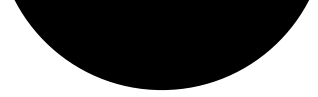

Pandora searches the world literature for evidence, news and other sources on matters of interest (doesn't shy awa from controversy) to br rsy) to bring to the reader. She welcomes comments and suggestions (via ip@rcpsych. ac.uk)

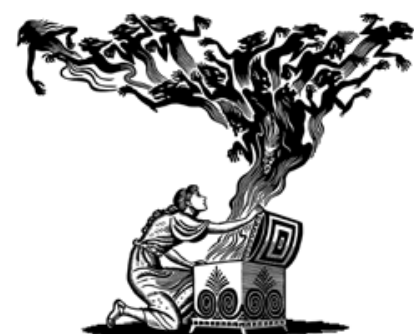

Can we predict whether our patients will respond to the antipsychotic drug prescribed?

$\mathrm{W}$ e know that not all patients respond to our treatments but our ability to predict the response has so far been limited to the use of clinical indicators and trial and error. Investigators at the Feinstein Institute claim that it is now possible to predict antipsychotic drug response using functional magnetic resonance imaging (fMRI). The researchers used the connectivity patterns of the striatum (which tends to be atypical in patients with psychotic disorders) to create an index. They carried out fMRI scans in patients experiencing a first episode of psychosis, before treatment, and found that the striatum connectivity pattern index significantly predicted whether psychotic symptoms would decrease after treatment. The authors strengthened the significance of their findings by applying this index also to a group of patients with a chronic psychotic illness, and demonstrating its equally predictive value.

Sarpal, D. K., Argyelan, M., Robinson, D. G., et al (2015) Baseline striatal functional connectivity as a predictor of response to antipsychotic drug treatment. American Journal of Psychiatry (doi: 10.1176/appi.ajp.2015.14121571).

Do antipsychotic drugs affect brain structure and does class of drug matter?

T nlike lithium, which increases the thickness of grey matter, antipsychotic drugs appear to decrease this. MRI studies have shown progressive loss of grey matter, which is attributed to the duration of antipsychotic drug treatment or cumulative antipsychotic use.

A recently published study confirmed these findings but went one step further in trying to distinguish between first- and second-generation antipsychotics. In a meta-analysis and metaregression of longitudinal MRI studies, the authors found that, unlike with first-generation antipsychotics, patients treated only with secondgeneration drugs showed less progressive loss of brain cortical grey matter. Could this mean that the second-generation antipsychotics have a neuroprotective effect? More work is needed to clarify the mechanisms involved in the progression of structural changes in the brain before we are able to draw conclusions about the effects of drugs and possible differences in class effects.

Vita, A., De Peri, L., Deste, G., et al (2015) The effect of antipsychotic treatment on cortical gray matter changes in schizophrenia: does the class matter? Biological Psychiatry, 78, 403 (doi: 10.1016/j.biopsych.2015.02.008).

\section{Effective treatment for Alzheimer's disease?}

Drecision medicine is here to stay, as it can lead to clinically useful developments. Several drugs are available for the treatment of Alzheimer's disease but the results are poor; at best they may modestly slow down the process. Could nerve growth factor (NGF), a protein essential to the growth and survival of neuronal cells, be the answer?

In a phase I study, NGF was injected directly into the brains of a small number of patients with
Alzheimer's disease. In a post-mortem examination of the brains of ten of the patients who had had this treatment (who died $1-10$ years after taking part in the study), the authors found that the neurons affected by Alzheimer's disease showed increased growth, axonal sprouting and activation of functional markers. The results of the phase II study, yet to be published, will show whether these anatomical changes are associated with clinical improvement.

Tuszynski, M. H., Yang, J. H., Barba, D., et al (2015) Nerve growth factor gene therapy. JAMA Neurology (doi: 10.1001/ jamaneurol.2015.1807).

\section{Adverse effects of antidepressants in children and adolescents}

ff-licence prescribing of antidepressants in children and adolescents came to an end a few years ago when it came to light that they can increase suicidality in the young. A recent systematic review and meta-analysis of reports from 70 trials of antidepressant drugs examined suicidality as well as aggressive behaviour in over 18000 patients (adults and children and adolescents), treated with selective serotonin reuptake inhibitors (SSRIs) and serotonin-noradrenaline reuptake inhibitors (SNRIs). While in adults there was no significant increase in either of these indices during treatment compared with placebo, in children and adolescents there was a doubling of the risk of suicidality and aggression. This confirms previous evidence as far as suicidality is concerned but the increase in aggression is a new finding and it does raise concerns.

Sharma, T., Guski, L. S., Freund, N., et al (2016) Suicidality and aggression during antidepressant treatment: systematic review and meta-analyses based on clinical study reports. BMJ, 352 (doi: http:// dx.doi.org/10.1136/bmj.i65).

\section{Euthanasia for people with a mental illness?}

$\mathrm{T}$ the last issue, Pandora touched on the subject of euthanasia and assisted suicide (EAS). Having opened the box, it has not been possible to put the lid back on as yet. A recent publication by Kim et al raises major ethical questions for psychiatrists across the globe to consider.

Several states in the USA and some European countries have some form of legislation that allows assisted suicide for people who are terminally ill. Belgium and the Netherlands have extended this to include people with mental disorders. However, little is known about how this practice is regulated in these countries. EAS have been practised for several decades in the Netherlands, although formal legislation was not introduced until 2002, with the Termination of Life on Request and Assisted Suicide - Review Procedures Act. In accordance with that Act there are regional euthanasia review committees, which review all instances of EAS and reports as to whether the notifying physicians (physicians of record for the performance of EAS) have conformed with the due care criteria laid out in the legislation. These are specially trained doctors (mostly general practitioners but also some psychiatrists), called 'support 
and consultation on euthanasia in the Netherlands' (SCEN) physicians. They usually serve as the official independent physician EAS consultants but they can also dispense less formal advice and assistance. The due care criteria set out in the Dutch EAS Act determine that the EAS physician is required to ensure that:

- the patient's request is voluntary and well considered

- the patient's suffering is unbearable and there is no prospect of improvement

- the patient has been informed about the situation and the prognosis

- there is no reasonable alternative in the patient's situation

- the patient has consulted at least one other, independent physician, who must see the patient and give a written opinion on whether the due care criteria set out above have been fulfilled

- due medical care and attention have been exercised in terminating the patient's life or assisting in suicide.

Kim and colleagues reviewed 66 psychiatric EAS cases from 2011 to 2014. The findings raise many questions. Seventy per cent of the patients were women and a quarter were 30-50 years old. Most patients had chronic, severe conditions and a history of attempted suicide; most were thought to have personality disorders and were described as socially isolated and lonely. There were also cases of psychosis, post-traumatic stress disorder, anxiety, somatoform disorders, eating disorders, as well as neurocognitive states, autism and prolonged grief. A quarter of the patients had EAS delivered by a physician new to them and although consultation with other physicians was extensive, in one-fifth of the cases there had been no independent psychiatric input, while in a quarter of the cases there was disagreement among consultants. In one case the euthanasia review committee found that it had failed to meet legal due care criteria.
The study findings raise many questions. How effectively are the criteria applied? How is capacity assessed? How can the physicians be so confident that there is no prospect for improvement in an individual's mental disorder? We still do not have clear and definitive indicators of outcome in these conditions. Most of the EAS patients had a diagnosis of depression, which inevitably would negatively influence their evaluations of their lives and future. Many people in the study felt lonely and isolated; in an affluent European country, couldn't there be psychosocial interventions to help improve people's quality of life? Most of these people were women. More questions can be raised but Pandora asks the fundamental question: is this really ethical?

Kim, S. Y. H., De Vries, R. G. \& Peteet, J. R. (2016) Euthanasia and assisted suicide of patients with psychiatric disorders in the Netherlands 2011 to 2014. JAMA Psychiatry (doi:10.1001/ jamapsychiatry.2015.2887)

\section{No health without mental health}

$\mathrm{M}$ ental health funding, both for research and service provision, has always lagged behind that for physical health and the global economic crisis, in recent years, has widened the gap. However, there is a glimpse of hope on the horizon. An initiative led by the World Health Organization may bring about a much needed change to the current state of affairs. A meeting is due to be held in April 2016, in Washington, DC, co-hosted by the World Bank Group and the World Health Organization. The meeting's aims are:

- to move mental health from the margins to the mainstream of the global health agenda by raising its profile

- to present the case for investing in mental health

- to identify entry points for renewed action and investment at the country, regional and global levels

- to mobilise a global coalition for action for scaled up implementation of mental health services for depression and anxiety in primary care and community settings.

\section{Asylum Seekers and Refugees Mental Health Network}

The Faculty of General Adult Psychiatry of the Royal College of Psychiatrists has launched seven networks, one of which is the Asylum Seekers and Refugees Mental Health Network. This network will allow interested psychiatrists to communicate with other colleagues in psychiatry through the sharing of information, knowledge and peer support. Its aims are:

- to allow psychiatrists working with asylum seekers and refugees to maintain an awareness of this changing and evolving area

- to provide a source of information on resources for this patient group

- to share information on the latest evidence base for treatment

- to offer peer support, allowing psychiatrists to share accounts of their clinical experience with this group.

The network leads are Dr Piyal Sen and Dr Katy Briffa. If you are interested in joining this network please contact the Faculty of General Adult Psychiatry Committee Manager, Lauren Wright, at lauren.wright@rcpsych.ac.uk. 